\title{
Adherence to a Mediterranean diet and risk of diabetes: a systematic review and meta-analysis
}

\author{
Lukas Schwingshack**, Benjamin Missbach, Jürgen König and Georg Hoffmann \\ University of Vienna, Faculty of Life Sciences, Department of Nutritional Sciences, Althanstraße 14 UZA II, A-1090 \\ Vienna, Austria
}

Submitted 27 November 2013: Final revision received 10 June 2014: Accepted 23 June 2014: First published online 13 August 2014

\begin{abstract}
Objective: Adherence to a Mediterranean diet is associated with significant improvements in health status. However, to date no systematic review and metaanalysis has summarized the effects of Mediterranean diet adherence on the risk of type 2 diabetes mellitus.

Design: Electronic searches for randomized controlled trials and cohort studies were performed in MEDLINE, SCOPUS, EMBASE and the Cochrane Trial Register until 2 April 2014. Pooled effects were calculated by an inverse-variance randomeffect meta-analysis using the statistical software Review Manager $5 \cdot 2$ by the Cochrane Collaboration.

Setting: Meta-analysis of randomized controlled trials and cohort studies.

Subjects: Eligibility criteria: 19+ years of age.

Results: One randomized controlled trial and eight prospective cohort studies (122810 subjects) published between 2007 and 2014 were included for metaanalysis. For highest $v$. lowest adherence to the Mediterranean diet score, the pooled risk ratio was 0.81 (95\% CI $\left.0.73,0.90, P<0.0001, I^{2}=55 \%\right)$. Sensitivity analysis including only long-term studies confirmed the results of the primary analysis (pooled risk ratio $=0.75$; $95 \%$ CI $0.68,0.83, P<0.00001, I^{2}=0 \%$ ). The Egger regression test provided no evidence of substantial publication bias $(P=0 \cdot 254)$.

Conclusions: Greater adherence to a Mediterranean diet is associated with a significant reduction in the risk of diabetes (19\%; moderate quality evidence). These results seem to be clinically relevant for public health, in particular for encouraging a Mediterranean-like dietary pattern for primary prevention of type 2 diabetes mellitus.
\end{abstract}

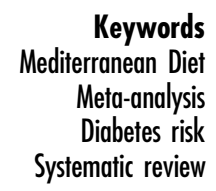

The worldwide incidence of people with type 2 diabetes mellitus (T2DM) is projected to rise from 171 million in 2000 to 380 million in 2025, causing enormous costs for health-care systems ${ }^{(1)}$. The typical Mediterranean diet (MD), which was first postulated by Ancel Keys in the $1960 s^{(2)}$, is characterized by high intakes of MUFA, vegetables and fruits, plant proteins, whole grains, fish and low-fat dairy products, moderate alcohol (red wine) intake, and low red meat consumption ${ }^{(3)}$. In the Seven Countries Study, Keys reported the beneficial effects of an MD on human health ${ }^{(2)}$. Since then, dietary pattern analysis has been widely used to detect the cumulative effects of diet on health. T2DM is a major chronic disease originating from different genetic, behavioural and environmental risks ${ }^{(4)}$, with impaired glucose tolerance being an indicator for the development of a diabetic metabolic state ${ }^{(5)}$. Lifestyle intervention studies in randomized trial settings have demonstrated a reduction in the relative risk to develop T2DM of $30-67 \%{ }^{(6)}$ with sustainable long-term reduction of incidence ${ }^{(7,8)}$, although a need to develop standardized lifestyle interventions may be necessary to enhance their efficacy ${ }^{(9)}$. MD is a homogeneous and straightforward construct having its roots in southern European eating patterns. Adherence to an MD is associated with significant improvements in health status ranging from reduced risk of mortality to neurodegenerative diseases ${ }^{(10,11)}$. However, to date no systematic review and meta-analysis has summarized the effects of 
MD adherence on the risk of T2DM. Therefore, the aim of the present study was to meta-analyse randomized controlled trials and cohort studies that have assessed the effect of adherence to an MD on the risk of T2DM.

\section{Methods}

\section{Literature search}

Literature searches were performed using the electronic databases MEDLINE, SCOPUS, EMBASE and the Cochrane Trial Register until 2 April 2014 using the following keywords: 'Mediterranean diet AND diabetes'. Moreover, the reference lists from retrieved articles were checked to search for further relevant studies, and systematic reviews and meta-analysis were searched by two authors. The literature search as well as article abstraction was conducted independently by two authors (L.S. and B.M.). Relevance of studies was assessed with a hierarchical approach (title, abstract and full manuscript). After an initial screening of titles and abstracts, studies included by both reviewers were compared and disagreement was resolved by consensus.

\section{Eligibility criteria}

Studies were included in the meta-analysis if they met all of the following criteria: (i) randomized controlled trial or cohort study design; (ii) reported adjusted relative risks (RR) for T2DM with corresponding 95\% confidence intervals; (iii) evaluated the association of an a priori score used for assessing adherence to an MD; and (iv) when a study seemed to have been published in duplicate, the version containing the most comprehensive information was selected. Studies including pregnant women were excluded, as were case-control studies, cross-sectional studies and studies that were not published as original reports (such as conference abstracts, letters to the editor without original data, commentaries and reviews).

\section{Quality assessment}

Full copies of studies were independently assessed for methodological quality by two authors (L.S. and B.M.) using the Jadad score for randomized controlled trials and the Newcastle Ottawa Scale for cohort studies ${ }^{(12,13)}$ (Table 1).

\section{Data extraction}

Two investigators (L.S. and B.M.) independently collected the relevant reports using a standardized form. The following data were extracted from each study: (i) first author's last name; (ii) year of publication; (iii) country of origin; (iv) sample size; (v) study length; (vi) age at entry; (vii) sex; (viii) T2DM diagnosis criteria; (ix) study design; (x) components of the MD score; (xi) adjustment factors; (xii) quality score; (xiii) dietary assessment method; and (xiv) risk estimates with their corresponding 95\% confidence intervals. When a study provided several risk estimates, the maximally adjusted models were chosen.

\section{Statistical analysis}

The meta-analysis was performed with the adjusted RR of the highest compared with the lowest MD adherence score based on random-effects modelling according to DerSimonian and Laird incorporating both within- and between-study variability. To evaluate the weighting of each study, the se for $\log (\mathrm{RR})$ of each study was calculated and regarded as the estimated variance of $\log (\mathrm{RR})^{(14)}$. Random-effects modelling with restricted maximum likelihood estimate, which is more appropriate if the number of included studies is small, was also used to confirm the final risk estimates. Heterogeneity was estimated by the Cochrane $Q$ test together with the $I^{2}$ statistic. An $I^{2}$ value $>50 \%$ indicates substantial heterogeneity across studies ${ }^{(15)}$. Funnel plots were used to assess potential publication bias. To determine the presence of publication bias, the symmetry of the funnel plots in which mean differences were plotted against their corresponding se was assessed. In addition, the Egger test was performed to test for potential publication bias $^{(16)}$.

\section{Results}

\section{Literature search}

Nine papers extracted from 1408 articles met the eligibility criteria and were included in the quantitative analysis ${ }^{(17-25)}$. One cohort study was excluded since the primary outcome was the risk of gestational diabetes ${ }^{(26)}$. The detailed steps of the meta-analysis article selection process are described as a flow diagram in Fig. 1.

\section{Study characteristics}

One randomized controlled trial and eight prospective cohort studies (sample size: 122810 subjects) published between 2007 and 2014 were included for meta-analysis. Study duration plus follow-up ranged between 3.2 and 20 years. All cohort studies used an FFQ for dietary assessment. General study characteristics are summarized in Table 1.

To assess the quality of the included studies, a 9-point scoring system was used according to the Newcastle Ottawa Scale. A score of $\geq 7$ points was defined as high study quality study (see online supplementary material, Table S1); all cohort studies were of high quality. The cut-offs for the high $v$. low MD score differed between the studies (three cohorts used quintiles ${ }^{(17,18,20)}$, two studies used quartiles ${ }^{(23,26)}$ and tertiles ${ }^{(19,22)}$, and one study used cluster analysis $\left.{ }^{(21)}\right)$.

\section{Mediterranean diet and risk of diabetes}

The pooled RR for the highest $v$. lowest adherence to the MD score was 0.81 (95\% CI 0.73, 0.90, P<0.0001, $I^{2}=55 \%$; Fig. 2). Stratified RR are summarized in Table S2 (see online supplementary material). For studies with a longterm follow-up ( $\geq 10$ years), an RR of 0.75 (95\% CI 0.68 , 
Table 1 General characteristics of the studies included in the present meta-analysis

\begin{tabular}{|c|c|c|c|c|c|c|c|c|c|c|}
\hline $\begin{array}{l}\text { Authors, year } \\
\text { and } \\
\text { reference }\end{array}$ & $\begin{array}{l}\text { Country and } \\
\text { cohort name }\end{array}$ & $\begin{array}{l}\text { Study } \\
\text { type }\end{array}$ & $\begin{array}{l}\text { Outcome and } \\
\text { criteria }\end{array}$ & $\begin{array}{l}\text { Population and } \\
\text { health status }\end{array}$ & $\begin{array}{l}\text { Follow-up } \\
\text { (years) }\end{array}$ & $\begin{array}{l}\text { Age at } \\
\text { entry } \\
\text { (years) }\end{array}$ & Sex & $\begin{array}{l}\text { Components of MD score or } \\
\text { dietary intervention }\end{array}$ & Adjustments & $\begin{array}{l}\text { Quality } \\
\text { score }\end{array}$ \\
\hline $\begin{array}{l}\text { Abiemo et al. } \\
(2013)^{(17)}\end{array}$ & $\begin{array}{l}\text { USA } \\
\text { Multi-Ethnic } \\
\text { Study of } \\
\text { Atherosclerosis } \\
\text { (MESA) }\end{array}$ & Cohort & $\begin{array}{l}\text { T2DM } \\
\text { FPG }<7.0 \mathrm{mmol} / \mathrm{l} ; \\
\text { fasting serum } \\
\text { insulin levels }\end{array}$ & $\begin{array}{l}5390 \\
\text { At the baseline } \\
\text { exam free of } \\
\text { clinical CVD }\end{array}$ & 6 & $45-84$ & $\mathrm{M} / \mathrm{W}$ & $\begin{array}{l}\text { (i) } \uparrow \text { Vegetables (except } \\
\text { potatoes); (ii) } \uparrow \text { legumes; } \\
\text { (iii) } \uparrow \text { fruit and fruit juices; } \\
\text { (iv) } \uparrow \text { nuts; (v) } \uparrow \text { whole } \\
\text { grain products; (vi) } \uparrow \text { fish; } \\
\text { (vii) } \downarrow \text { red meat and } \\
\text { processed meats; } \\
\text { (viii) } \uparrow \text { MUFA:SFA; } \\
\text { (ix) } \downarrow \text { dairy products than } \\
\text { median intake }\end{array}$ & $\begin{array}{l}\text { Age, sex, race/ethnicity, } \\
\text { educational level, } \\
\text { family income, PA, } \\
\text { smoking status, total } \\
\text { energy intake, WC }\end{array}$ & 7 \\
\hline $\begin{array}{l}\text { Brunner et al. } \\
\quad(2008)^{(21)}\end{array}$ & $\begin{array}{l}\text { England } \\
\text { Whitehall II }\end{array}$ & Cohort & $\begin{array}{l}\text { T2DM } \\
\text { Self-report, } \\
\text { diabetic } \\
\text { medication, } \\
\text { OGTT }\end{array}$ & $\begin{array}{l}n 7731 \\
\text { At the baseline } \\
\text { exam free of } \\
\text { clinical CVD }\end{array}$ & 15 & $35-69$ & $\mathrm{M} / \mathrm{W}$ & $\begin{array}{l}\text { (i) } \uparrow \text { Wholemeal bread; } \\
\text { (ii) } \uparrow \text { fruits; (iii) } \uparrow \\
\text { vegetables; (iv) } \uparrow \text { pasta } \\
\text { and rice; (v) } \uparrow \text { wine; } \\
\text { (vi) } \downarrow \text { intake of full-cream } \\
\text { milk; (vii) } \uparrow \text { butter; } \\
\text { (viii) moderate white bread }\end{array}$ & $\begin{array}{l}\text { Age, sex, ethnicity, } \\
\text { dietary energy } \\
\text { misreporting, social } \\
\text { position, smoking } \\
\text { status, leisure-time } \\
\text { PA }\end{array}$ & 7 \\
\hline $\begin{array}{l}\text { De Koning } \\
\text { et al. } \\
\quad(2011)^{(18)}\end{array}$ & $\begin{array}{l}\text { USA } \\
\text { Health } \\
\text { Professionals } \\
\text { Follow Up } \\
\text { (HPFS) }\end{array}$ & Cohort & $\begin{array}{l}\text { T2DM } \\
\text { Self-report } \\
\text { medication use or } \\
\text { positive glucose } \\
\text { test (until 1998: } \\
\text { NDDG criteria; } \\
\text { after 1998: ADA } \\
\text { criteria; 97\% of } \\
\text { the cases were } \\
\text { validated by } \\
\text { medical record) }\end{array}$ & $\begin{array}{l}n 41615 \\
\text { At the baseline } \\
\text { exam free of } \\
\text { clinical CVD }\end{array}$ & $\leq 20$ & n.d. & $\mathrm{M}$ & $\begin{array}{l}\text { (i) } \uparrow \text { Vegetables (no } \\
\text { potatoes); (ii) } \uparrow \text { legumes; } \\
\text { (iii) } \uparrow \text { fruits; (iv) } \uparrow \text { nuts; } \\
\text { (v) } \uparrow \text { whole grains; } \\
\text { (vi) } \uparrow \text { fish; (vii) } \uparrow \text { MUFA: } \\
\text { SFA; (viii) } \downarrow \text { red meat and } \\
\text { processed meats; } \\
\text { (ix) moderate alcohol } \\
\text { intake }\end{array}$ & $\begin{array}{l}\text { Age, smoking, PA, } \\
\text { coffee intake, family } \\
\text { history of T2DM, BMI, } \\
\text { total energy }\end{array}$ & 9 \\
\hline $\begin{array}{l}\text { Salas-Salvado } \\
\text { et al. } \\
(2014)^{(25)}\end{array}$ & $\begin{array}{l}\text { Spain } \\
\text { Prevencion } \\
\text { con dieta } \\
\text { mediterranea } \\
\text { trial } \\
\text { (PREDIMED) }\end{array}$ & $\mathrm{RCT}$ & $\begin{array}{l}\text { T2DM } \\
\text { OGTT and FPG } \\
<7.0 \mathrm{mmol} / \mathrm{l}\end{array}$ & $\begin{array}{l}\text { n } 3541 \\
\text { At the baseline } \\
\text { exam free of } \\
\text { clinical CVD. At } \\
\text { least three of the } \\
\text { following CVD risk } \\
\text { factors: smoking, } \\
\text { hypertension, } \\
\text { dyslipidaemia, } \\
\text { overweight, family } \\
\text { history of } \\
\text { premature CVD }\end{array}$ & $4 \cdot 1$ & $55-80$ & $\mathrm{M} / \mathrm{W}$ & $\begin{array}{l}\text { (i) } \uparrow \text { Olive oil; (ii) } \uparrow \text { fruits, } \\
\text { vegetables, legumes and } \\
\text { fish; (iii) } \downarrow \text { total meat, } \\
\text { recommending white } \\
\text { meat instead of red or } \\
\text { processed meat; } \\
\text { (iv) homemade tomato } \\
\text { sauce; (v) } \downarrow \text { butter, cream, } \\
\text { fast food, sweets, pastries, } \\
\text { sugar-sweetened } \\
\text { beverages; (vi) moderate } \\
\text { red wine }\end{array}$ & $\begin{array}{l}\text { Energy intake, BMI, } \\
\text { WC, PA, smoking } \\
\text { status, FPG, use of } \\
\text { lipid-lowering drugs, } \\
\text { MedDiet Score }\end{array}$ & $4^{*}$ \\
\hline
\end{tabular}


Table 1 Continued

\begin{tabular}{|c|c|c|c|c|c|c|c|c|c|c|}
\hline $\begin{array}{l}\text { Authors, year } \\
\text { and } \\
\text { reference }\end{array}$ & $\begin{array}{l}\text { Country and } \\
\text { cohort name }\end{array}$ & $\begin{array}{l}\text { Study } \\
\text { type }\end{array}$ & $\begin{array}{l}\text { Outcome and } \\
\text { criteria }\end{array}$ & $\begin{array}{l}\text { Population and } \\
\text { health status }\end{array}$ & $\begin{array}{l}\text { Follow-up } \\
\text { (years) }\end{array}$ & $\begin{array}{l}\text { Age at } \\
\text { entry } \\
\text { (years) }\end{array}$ & Sex & $\begin{array}{l}\text { Components of MD score or } \\
\text { dietary intervention }\end{array}$ & Adjustments & $\begin{array}{l}\text { Quality } \\
\text { score }\end{array}$ \\
\hline $\begin{array}{l}\text { InterAct } \\
\text { Consortium } \\
(2011)^{(22)}\end{array}$ & $\begin{array}{l}\text { European } \\
\text { Prospective Into } \\
\text { Cancer and } \\
\text { Nutrition (EPIC) } \\
\text { (excluded: } \\
\text { Greece, } \\
\text { Norway) }\end{array}$ & Cohort & $\begin{array}{l}\text { T2DM } \\
\text { Medical } \\
\text { record }\end{array}$ & $\begin{array}{l}n 16154 \\
\text { At the baseline } \\
\text { exam free of } \\
\text { clinical CVD }\end{array}$ & 4.4 & $25-70$ & $\mathrm{M} / \mathrm{W}$ & $\begin{array}{l}\text { (i) } \uparrow \text { Vegetables (no } \\
\text { potatoes); (ii) } \uparrow \text { legumes; } \\
\text { (iii) } \uparrow \text { fruits; (iv) } \uparrow \text { nuts; } \\
\text { (v) } \uparrow \text { whole grains; } \\
\text { (vi) } \uparrow \text { fish; (vii) } \uparrow \text { MUFA: } \\
\text { SFA; (viii) } \downarrow \text { red meat and } \\
\text { processed meats; (ix) } \\
\text { moderate alcohol intake }\end{array}$ & $\begin{array}{l}\text { Energy intake, sex, } \\
\text { BMI, education level, } \\
\text { PA, smoking status }\end{array}$ & 9 \\
\hline $\begin{array}{l}\text { Martínez- } \\
\text { González } \\
\text { et al. } \\
(2008)^{(19)}\end{array}$ & $\begin{array}{l}\text { Spain } \\
\text { Seguimento } \\
\text { Universidad de } \\
\text { Navarra (SUN) }\end{array}$ & Cohort & $\begin{array}{l}\text { T2DM } \\
\text { ADA (symptoms } \\
\text { of diabetes }+ \\
\text { random plasma } \\
\text { glucose } \\
\text { concentration } \\
\geq 11.1 \mathrm{mmol} / \mathrm{l} \text { or } \\
\text { FPG } \geq 7.0 \mathrm{mmol} / \mathrm{l}, \\
\text { OGTT) }\end{array}$ & $\begin{array}{l}n 13380 \\
\text { At the baseline } \\
\text { exam free of } \\
\text { clinical CVD }\end{array}$ & $3 \cdot 2$ & $20-29$ & $\mathrm{M} / \mathrm{W}$ & $\begin{array}{l}\text { (i) } \uparrow \text { Vegetables; } \\
\text { (ii) } \uparrow \text { legumes; (iii) } \uparrow \text { fruits; } \\
\text { (iv) } \uparrow \text { nuts; (v) } \uparrow \text { whole } \\
\text { grains; (vi) } \uparrow \text { fish; } \\
\text { (vii) } \uparrow \text { MUFA:SFA; } \\
\text { (viii) } \downarrow \text { meat and meat } \\
\text { products; (ix) moderate } \\
\text { milk and dairy products; } \\
\text { (x) moderate alcohol } \\
\text { intake }\end{array}$ & $\begin{array}{l}\text { Age, sex, total years of } \\
\text { university education, } \\
\text { BMl, family history of } \\
\text { diabetes, } \\
\text { hypertension at } \\
\text { baseline, PA, hours } \\
\text { sitting down per } \\
\text { week, smoking, total } \\
\text { energy intake }\end{array}$ & 8 \\
\hline $\begin{array}{l}\text { Mozaffarian } \\
\text { et al. } \\
(2007)^{(20)}\end{array}$ & $\begin{array}{l}\text { Italy } \\
\text { Gruppo Italiano } \\
\text { per lo Studio } \\
\text { della } \\
\text { sopravivenza } \\
\text { nell'infarto } \\
\text { miocardico } \\
\text { (GISSI) }\end{array}$ & Cohort & $\begin{array}{l}\text { T2DM } \\
\text { New use of } \\
\text { diabetic } \\
\text { medication or } \\
\text { FPG } \geq 7.0 \mathrm{mmol} / \mathrm{I}\end{array}$ & $\begin{array}{l}n 8291 \\
\quad \text { With MI }\end{array}$ & $11 \cdot 34$ & $20-90$ & $\mathrm{M} / \mathrm{W}$ & $\begin{array}{l}\text { (i) } \uparrow \text { Cooked and raw } \\
\text { vegetables; (ii) } \uparrow \text { fruits; } \\
\text { (iii) } \uparrow \text { fish; (iv) } \uparrow \text { olive oil; } \\
\text { (v) moderate alcohol } \\
\text { intake }\end{array}$ & $\begin{array}{l}\text { Age, sex, smoking } \\
\text { status, time from MI } \\
\text { to enrolment, } \\
\text { treatment } \\
\text { assignment, BMI, } \\
\text { maximum exercise } \\
\text { tolerance during } \\
\text { stress testing, } \\
\text { ischaemia during } \\
\text { stress testing, NYHA } \\
\text { heart failure } \\
\text { symptoms, CCS } \\
\text { angina symptoms, } \\
\text { history of } \\
\text { hypertension, prior MI } \\
\text { previous to index MI, } \\
\text { ACE inhibitor use, } \\
\text { ß-blocker use, } \\
\text { diuretic use, lipid- } \\
\text { lowering medication } \\
\text { use, consumption of } \\
\text { cheese, wine and } \\
\text { coffee }\end{array}$ & 7 \\
\hline
\end{tabular}




\begin{tabular}{|c|c|c|c|c|c|c|c|c|c|c|}
\hline $\begin{array}{l}\text { Authors, year } \\
\text { and } \\
\text { reference }\end{array}$ & $\begin{array}{l}\text { Country and } \\
\text { cohort name }\end{array}$ & $\begin{array}{l}\text { Study } \\
\text { type }\end{array}$ & $\begin{array}{l}\text { Outcome and } \\
\text { criteria }\end{array}$ & $\begin{array}{l}\text { Population and } \\
\text { health status }\end{array}$ & $\begin{array}{l}\text { Follow-up } \\
\text { (years) }\end{array}$ & $\begin{array}{l}\text { Age at } \\
\text { entry } \\
\text { (years) }\end{array}$ & Sex & $\begin{array}{l}\text { Components of MD score or } \\
\text { dietary intervention }\end{array}$ & Adjustments & $\begin{array}{l}\text { Quality } \\
\text { score }\end{array}$ \\
\hline $\begin{array}{l}\text { Rossi et al. } \\
\qquad(2013)^{(23)}\end{array}$ & $\begin{array}{l}\text { European } \\
\text { Prospective Into } \\
\text { Cancer and } \\
\text { Nutrition (EPIC) } \\
\text { (only Greek } \\
\text { cohort) }\end{array}$ & Cohort & $\begin{array}{l}\text { T2DM } \\
\text { Self-report, } \\
\text { medical record, } \\
\text { diabetic } \\
\text { medication use }\end{array}$ & $\begin{array}{l}n 22295 \\
\text { At the baseline } \\
\text { exam free of } \\
\text { clinical CVD }\end{array}$ & 4 & 20-80 & $\mathrm{M} / \mathrm{W}$ & $\begin{array}{l}\text { (i) } \uparrow \text { Vegetables; } \\
\text { (ii) } \uparrow \text { legumes; (iii) } \uparrow \text { fruits; } \\
\text { (iv) } \uparrow \text { nuts; (v) } \uparrow \text { cereals; } \\
\text { (vi) } \uparrow \text { fish and seafood; } \\
\text { (vii) } \uparrow \text { MUFA:SFA; } \\
\text { (viii) } \downarrow \text { meat and meat } \\
\text { products; (ix) } \downarrow \text { dairy } \\
\text { products; (x) } 10-50 \mathrm{~g} \\
\text { ethanol/d for men and } \\
5-25 \mathrm{~g} \text { ethanol/d for } \\
\text { women considered as } \\
\text { moderate alcohol } \\
\text { consumption }\end{array}$ & $\begin{array}{l}\text { Age, sex, level of } \\
\text { education, PA, BMI, } \\
\text { WHR, total energy } \\
\text { intake }\end{array}$ & 9 \\
\hline $\begin{array}{l}\text { Tobias et al. } \\
\qquad(2012)^{(24)}\end{array}$ & $\begin{array}{l}\text { USA } \\
\text { Nurses' Health } \\
\text { Study II (NHS II) }\end{array}$ & Cohort & $\begin{array}{l}\text { T2DM } \\
\text { One or more } \\
\text { classic symptoms } \\
\text { (i.e. excessive } \\
\text { thirst, polyuria, } \\
\text { etc.) and FPG } \\
<7.7 \mathrm{mmol} / / \text { (after } \\
\text { 1998: }<7.0 \mathrm{mmol} / /) \\
\text { or random plasma } \\
\text { glucose level } \\
\geq 11 \mathrm{mmol} / \mathrm{l}\end{array}$ & $\begin{array}{l}n 4413 \\
\text { With prior } \\
\text { GDM }\end{array}$ & 14 & $24-44$ & W & $\begin{array}{l}\text { (i) } \uparrow \text { Vegetables; } \\
\text { (ii) } \uparrow \text { legumes and soya; } \\
\text { (iii) } \uparrow \text { fruits; (iv) } \uparrow \text { nuts; } \\
\text { (v) } \uparrow \text { whole grain products; } \\
\text { (vi) } \uparrow \text { fish and seafood; } \\
\text { (vii) } \uparrow \text { MUFA:SFA; } \\
\text { (viii) } \downarrow \text { red meat and } \\
\text { processed meats; (ix) } \\
\text { moderate alcohol intake }\end{array}$ & $\begin{array}{l}\text { Age, total energy intake, } \\
\text { age at first birth, } \\
\text { parity, ethnicity, } \\
\text { parental diabetes, } \\
\text { oral contraceptive } \\
\text { use, menopause, } \\
\text { smoking status }\end{array}$ & 8 \\
\hline
\end{tabular}

MD, Mediterranean diet; RCT, randomized controlled trial; FPG, fasting plasma glucose; OGGT, oral glucose tolerance test; NDDG, National Diabetes Data Group; ADA, American Diabetes Association; MI, myocardial infarction; GDM, gestational diabetes mellitus; n.d., not determined; M, men, W, women; PA, physical activity; WC, waist circumference; NYHA, New York Heart Association; CCS, Canadian Cardiovascular Society; ACE,

ADA criteria: symptoms of diabetes plus casual plasma glucose concentration $\geq 11.1 \mathrm{mmol} / \mathrm{l}$ or FPG $\geq 7.0 \mathrm{mmol} / \mathrm{l}$, or $2 \mathrm{~h}$ post-load glucose $\geq 11.1 \mathrm{mmol} / \mathrm{l}$ during an $\mathrm{OGGT}$; in the absence of unequivocal hyperglycaemia. Jadad score. 


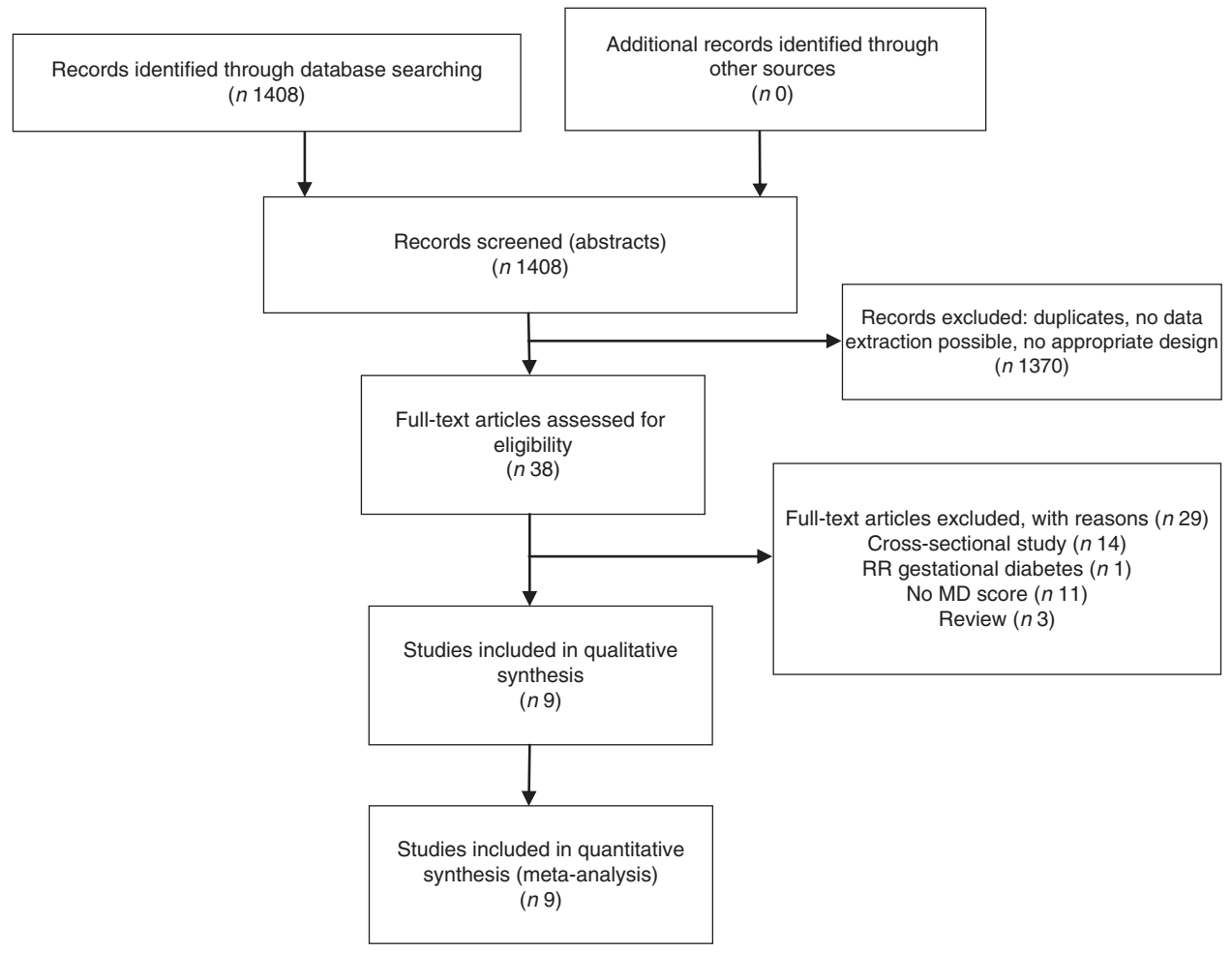

Fig. 1 Flow diagram showing detailed steps of the article selection process for the present meta-analysis (RR, relative risk; MD, Mediterranean diet)

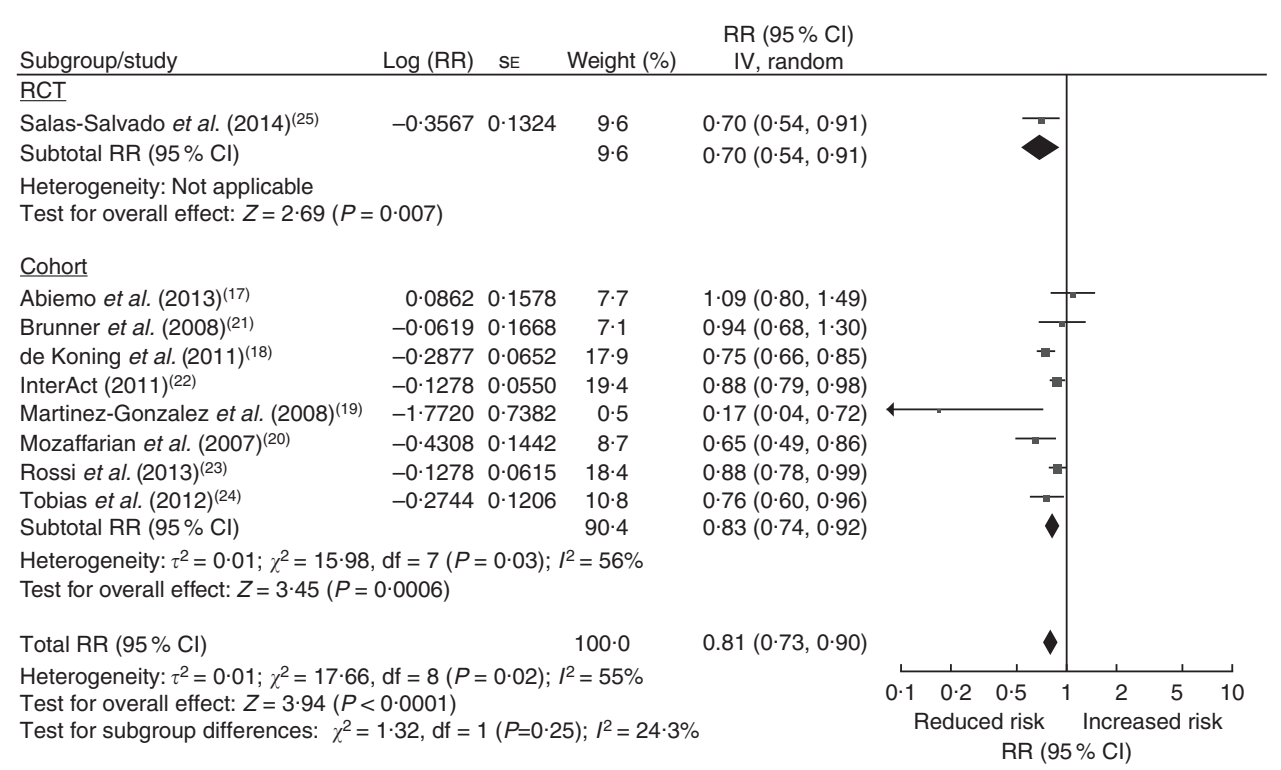

Fig. 2 Forest plot showing the relative risk (RR) and $95 \%$ confidence interval of type 2 diabetes for the highest $v$. lowest adherence to a Mediterranean diet score for one randomized controlled trail (RCT) and eight prospective cohort studies (122810 subjects) published between 2007 and 2014. For each study, the black square indicates the RR, the size of which indicates the study's weight in the analysis (weights are from random-effects analysis) and the horizontal line represents the $95 \% \mathrm{Cl}$. The centre of the diamond indicates the summary estimate of the RR and its width represents the $95 \% \mathrm{Cl}$ of the summary RR estimate

0.83, $\left.P<0 \cdot 00001, I^{2}=0 \%\right)$ was found, and for large sample size ( $\geq 10000)$, an RR of 0.82 (95\% CI 0.72, 0.94, $P=0.005$, $I^{2}=67 \%$ ) could be observed. There was a significant association in the European analysis $(\mathrm{RR}=0.81 ; 95 \% \mathrm{CI}$
$0.71,0.93, P=0.002)$ but not in the US analysis ( RR $=0.82$; $95 \%$ CI $0 \cdot 68,1 \cdot 00, P=0 \cdot 05)$. Exclusion of studies by Tobias et al. $^{(24)}$ (history of gestational diabetes) and Mozaffarian et $a{ }^{(20)}$ (secondary prevention of CVD) confirmed the 
results of the main analysis $(\mathrm{RR}=0.84 ; 95 \% \mathrm{CI} 0.75$, 0.94, $\left.P=0.003, I^{2}=57 \%\right)$. Since cut-off and MD scores varied between studies, we performed a sensitivity analysis investigating the association between a 2-point increase of adherence to the MD score (defined by Trichopoulou ${ }^{(27)}$ ) and the risk of T2DM. Six of nine ${ }^{(17-19,22,23,26)}$ studies were included and showed a significant risk reduction $\left(\mathrm{RR}=0.93 ; 95 \%\right.$ CI $0.89,0.98, P=0.007, I^{2}=69 \%$; see online supplementary material, Fig. S1).

\section{Publication bias}

The funnel plots for diabetes incidence indicated low asymmetry (with the exception of one outlier), suggesting that the risk of publication bias is low (see online supplementary material, Fig. S2). The Egger test for small study effects yielded non-significant results $(P=0 \cdot 254)$.

\section{Discussion}

The present meta-analysis including $>100000$ subjects revealed a significant association between adherence to dietary patterns exhibiting specific MD characteristics and decreased risk of T2DM. The overall quality of evidence rated according to the GRADE guidelines for the main outcome was moderate ${ }^{(28)}$. To the best of our knowledge, the present study represents the first meta-analysis showing a possible association between adherence to an MD and risk of T2DM.

With respect to potential mechanisms of action, there seems to be a causal link between oxidative stress, inflammation, endothelial dysfunction and diabetes ${ }^{(29)}$. Interconnections between MD and markers of inflammation/ endothelial function have been suggested by a recent metaanalysis of intervention trials ${ }^{(30)}$. Different adherence scores measuring compliance to an MD were used in epidemiological studies since Keys' first findings, and one of the most relevant scores was established by Trichopoulou et al. ${ }^{(27)}$. Several studies of the present meta-analysis used MD scores based on Trichopoulou et al. with only slight variations. The combination of components and the adherence to the whole concept of an MD seems pivotal: increasing fruit and vegetable intake as a sole option only showed weak overall effects on risk for developing $\mathrm{T}_{2} \mathrm{DM}^{(31)}$. Making use of synergistic effects of different nutrient compounds especially in an MD has shown to be more distinct and effective. Thus, compared with targeting single nutrients on their health impact, dietary pattern analysis is especially useful in a public health context providing clear-cut messages for stakeholders.

In our meta-analysis, the relative risk for developing T2DM when adhering to an MD was significantly different when comparing European and US studies. Risk for T2DM development was significantly more decreased in European studies as compared with their US counterparts. This finding may be due to confounders that were not assessed in the various studies. Subgroup analysis including studies of great population (sample size $\geq 10000$ ) and long-term follow-up ( $\geq 10$ years) resulted in a more pronounced effect of MD adherence on T2DM (RR $=0.82$, $P=0.005, I^{2}=67 \%$ and $\left.\mathrm{RR}=0.75, P<0.00001, I^{2}=0 \%\right)$ when compared with studies with a smaller sample size $(<10000)$ or short-term studies ( $<10$ years). Especially long-term follow-up showed a prominent and significant decrease of T2DM risk of $25 \%$, suggesting a beneficial longterm effect of MD on T2DM risk.

There are some limitations to our study that should be considered. First, although most studies used a stringent MD score computation based on Trichopoulous et al. ${ }^{(27)}$, a more homogeneous MD score computation would be desirable to make studies even more comparable. Second, two studies with a slightly different study and population design (Mozaffarian et al., secondary prevention of $\mathrm{CVD}^{(20)}$; Tobias et al., volunteers with history of gestational diabetes ${ }^{(24)}$ ) were included in the present meta-analysis. Thus, all subjects in these studies had a history of a disease, which may be associated with higher diabetes risk than for healthy subjects. However, results of the primary analysis could be confirmed following exclusion of the respective studies. High inter-study heterogeneity most likely results in a reduction in reliability of estimates. Since considerable heterogeneity could be observed for the primary analysis in the present systematic review, additional sensitivity analyses were performed to evaluate whether this could have been due to a single study. However, no single study seemed to have a considerable effect on heterogeneity. Furthermore, meta-analyses are limited by the included study types and cohort studies are at risk of various potential sources of bias. For example, response bias as well as errors in the assessment of diets is a persistent problem when analysing associations between diet and disease.

\section{Conclusion}

In conclusion, the current systematic review and metaanalysis shows that adherence to an MD is associated with a decreased risk of becoming diabetic at a reasonable magnitude (19\%). Overall, the present study supports the relevancy and effectiveness of a long-term adherence to an $\mathrm{MD}$ as an important measure in the primary prevention of T2DM.

\section{Acknowledgements}

Acknowledgements: The authors are grateful to Francesco Sofi (MD, PhD) for statistical advice. Financial support: This research received no specific grant from any funding agency in the public, commercial or not-for-profit sectors. Conflict of interest: None. Authorship: L.S. and B.M. conducted the data analysis; L.S., B.M., J.K. and G.H. interpreted the 
results, drafted the manuscript and finalized the manuscript. All authors read and approved the final manuscript. Ethics of buman subject participation: Ethical approval was not required.

\section{Supplementary material}

To view supplementary material for this article, please visit http://dx.doi.org/10.1017/S1368980014001542

\section{References}

1. Hossain P, Kawar B \& El Nahas M (2007) Obesity and diabetes in the developing world - a growing challenge. $N$ Engl J Med 356, 213-215.

2. Keys A, Menotti A, Karvonen MJ et al. (1986) The diet and 15-year death rate in the seven countries study. Am J Epidemiol 124, 903-915.

3. Willett WC, Sacks F, Trichopoulou A et al. (1995) Mediterranean diet pyramid: a cultural model for healthy eating. Am J Clin Nutr 61, 6 Suppl., 1402S-1406S.

4. Neel JV (1962) Diabetes mellitus: a 'thrifty' genotype rendered detrimental by 'progress'? Am J Hum Genet 14, 353-362.

5. Group TDS (1999) Glucose tolerance and mortality: comparison of WHO and American Diabetes Association diagnostic criteria. The DECODE study group. European Diabetes Epidemiology Group. Diabetes Epidemiology: Collaborative analysis of Diagnostic criteria in Europe. Lancet 354, 617-621.

6. Yamaoka K \& Tango T (2005) Efficacy of lifestyle education to prevent type 2 diabetes: a meta-analysis of randomized controlled trials. Diabetes Care 28, 2780-2786.

7. Lindstrom J, Ilanne-Parikka P, Peltonen M et al. (2006) Sustained reduction in the incidence of type 2 diabetes by lifestyle intervention: follow-up of the Finnish Diabetes Prevention Study. Lancet 368, 1673-1679.

8. Li G, Zhang P, Wang J et al. (2008) The long-term effect of lifestyle interventions to prevent diabetes in the China Da Qing Diabetes Prevention Study: a 20-year follow-up study. Lancet 371, 1783-1789.

9. Yoon U, Kwok LL \& Magkidis A (2013) Efficacy of lifestyle interventions in reducing diabetes incidence in patients with impaired glucose tolerance: a systematic review of randomized controlled trials. Metabolism 62, 303-314.

10. Sofi F, Abbate R, Gensini GF et al. (2010) Accruing evidence on benefits of adherence to the Mediterranean diet on health: an updated systematic review and meta-analysis. Am J Clin Nutr 92, 1189-1196.

11. Schwingshackl L \& Hoffmann G (2014) Adherence to Mediterranean diet and risk of cancer: a systematic review and meta-analysis of observational studies. Int $J$ Cancer (Epublication ahead of print version).

12. Jadad AR, Moore RA, Carroll D et al. (1996) Assessing the quality of reports of randomized clinical trials: is blinding necessary? Control Clin Trials 17, 1-12.

13. Wells GA, Shea B, O'Connell D et al. (not dated) The Newcastle-Ottawa Scale (NOS) for assessing the quality of nonrandomised studies in meta-analyses. http://www.ohri. ca/programs/clinical_epidemiology/oxford.htm (accessed October 2013).
14. DerSimonian R \& Laird N (1986) Meta-analysis in clinical trials. Control Clin Trials 7, 177-188.

15. Higgins JP, Thompson SG, Deeks JJ et al. (2003) Measuring inconsistency in meta-analyses. BMJ 327, 557-560.

16. Egger M, Davey Smith G, Schneider M et al. (1997) Bias in meta-analysis detected by a simple, graphical test. BMJ $\mathbf{3 1 5}$, 629-634.

17. Abiemo EE, Alonso A, Nettleton JA et al. (2013) Relationships of the Mediterranean dietary pattern with insulin resistance and diabetes incidence in the Multi-Ethnic Study of Atherosclerosis (MESA). Br J Nutr 109, 1490-1497.

18. de Koning L, Chiuve SE, Fung TT et al. (2011) Diet-quality scores and the risk of type 2 diabetes in men. Diabetes Care 34, 1150-1156.

19. Martinez-Gonzalez MA, de la Fuente-Arrillaga C, NunezCordoba JM et al. (2008) Adherence to Mediterranean diet and risk of developing diabetes: prospective cohort study. BMJ 336, 1348-1351.

20. Mozaffarian D, Marfisi R, Levantesi G et al. (2007) Incidence of new-onset diabetes and impaired fasting glucose in patients with recent myocardial infarction and the effect of clinical and lifestyle risk factors. Lancet 370, 667-675.

21. Brunner EJ, Mosdol A, Witte DR et al. (2008) Dietary patterns and 15-y risks of major coronary events, diabetes, and mortality. Am J Clin Nutr 87, 1414-1421.

22. InterAct Consortium, Romaguera $\mathrm{D}$, Guevara $\mathrm{M}$ et al. (2011) Mediterranean diet and type 2 diabetes risk in the European Prospective Investigation into Cancer and Nutrition (EPIC) study: the InterAct project. Diabetes Care 34, 1913-1918.

23. Rossi M, Turati F, Lagiou P et al. (2013) Mediterranean diet and glycaemic load in relation to incidence of type 2 diabetes: results from the Greek cohort of the populationbased European Prospective Investigation into Cancer and Nutrition (EPIC). Diabetologia 56, 2405-2413.

24. Tobias DK, Hu FB, Chavarro J et al. (2012) Healthful dietary patterns and type 2 diabetes mellitus risk among women with a history of gestational diabetes mellitus. Arch Intern Med 172, 1566-1572.

25. Salas-Salvado J, Bullo M, Estruch R et al. (2014) Prevention of diabetes with Mediterranean diets: a subgroup analysis of a randomized trial. Ann Intern Med 160, 1-10.

26. Tobias DK, Zhang C, Chavarro J et al. (2012) Prepregnancy adherence to dietary patterns and lower risk of gestational diabetes mellitus. Am J Clin Nutr 96, 289-295.

27. Trichopoulou A, Costacou T, Bamia C et al. (2003) Adherence to a Mediterranean diet and survival in a Greek population. N Eng J Med 348, 2599-2608.

28. Guyatt G, Oxman AD, Akl EA et al. (2011) GRADE guidelines: 1. Introduction - GRADE evidence profiles and summary of findings tables. J Clin Epidemiol 64, 383-394.

29. Marin C, Yubero-Serrano EM, Lopez-Miranda J et al. (2013) Endothelial aging associated with oxidative stress can be modulated by a healthy Mediterranean diet. Int J Mol Sci $\mathbf{1 4}$, 8869-8889.

30. Schwingshackl L \& Hoffmann G (2014) Mediterranean dietary pattern, inflammation and endothelial function: a systematic review and meta-analysis of intervention trials. Nutr Metab Cardiovasc Dis (Epublication ahead of print version).

31. Cooper AJ, Forouhi NG, Ye Z et al. (2012) Fruit and vegetable intake and type 2 diabetes: EPIC-InterAct prospective study and meta-analysis. Eur J Clin Nutr 66, 1082-1092. 